\title{
Norois
}

Environnement, aménagement, société

$204 \mid 2007 / 3$

Patrimoine, culture et construction identitaire dans les territoires ruraux

\section{Motards, capital spatial et construction identitaire hétérotopique : récits et pérégrinations des motards rennais}

Bikers, spatial capital and heterotopic identity construction: stories and travels

of bikers from Rennes

\section{Valérie Delignières et Hervé Regnauld}

\section{OpenEdition}

Journals

Édition électronique

URL : http://journals.openedition.org/norois/1467

DOI : $10.4000 /$ norois. 1467

ISBN : 978-2-7535-1552-9

ISSN : $1760-8546$

Éditeur

Presses universitaires de Rennes

Édition imprimée

Date de publication : 1 septembre 2007

Pagination : 81-94

ISBN : 978-2-7535-0533-9

ISSN : 0029-182X

Référence électronique

Valérie Delignières et Hervé Regnauld, « Motards, capital spatial et construction identitaire

hétérotopique : récits et pérégrinations des motards rennais », Norois [En ligne], 204 | 2007/3, mis en ligne le 01 septembre 2009, consulté le 19 avril 2019. URL : http://journals.openedition.org/ norois/1467; DOI : 10.4000/norois. 1467 


\title{
MOTARDS, CAPITAL SPATIAL
}

ET CONSTRUCTION IDENTITAIRE HÉTÉROTOPIQUE : RÉCITS ET PÉRÉGRINATIONS DES MOTARDS RENNAIS

\author{
VALÉRIE DELIGNIÈRES \\ Costel - UMr LetG 6554 CNRS, IFR Caren, PRES Européen de Bretagne \\ (IUFM de Bretagne), \\ rue Saint-Malo - 35043 RenNES cedex \\ valerie.delignieres@bretagne.iufm.fr \\ HERVÉ RÉGNAULD \\ Costel - UMR LETG 6554 CNRS, IFR Caren, PRES Européen de Bretagne \\ (Université de Rennes 2 Haute-Bretagne), \\ place du Recteur-Henri-Le-Moal - 35043 Rennes cedex \\ herve.regnauld@uhb.fr
}

\begin{abstract}
RÉSUMÉ
Les motards de l'agglomération rennaise ont été interrogés sur leurs déplacements et leurs motivations. Leurs réponses ont été analysées dans leur dimension qualitative : elles constituent un discours cohérent dans lequel une identité motarde spécifique est revendiquée. Une étude quantitative des réponses montre en revanche que les déplacements des motards sont contraints par leur âge, leurs revenus, leur niveau social. Ils ne sont, à ce titre aucunement différents du reste de la population. L'écart entre le discours tenu et la pratique mesurée est ensuite interprété en terme de construction d'un capital spatial explicable avec le concept foucaldien d'hétérotopie.
\end{abstract}

Mots CLÉ : Capital spatial - Hétérotopie - Identité - Moto - Rennes.

ABSTRACT

Bikers, spatial capital and heterotopic identity construction: stories and travels of bikers from Rennes

In the vicinity of Rennes larger city, bikers have been asked to explain their moves and motivations. The qualitative part of their answers displays a well constructed address aimed at showing a strong peculiar identity. However, a quantitative approach of their movements shows that most of their travels are controlled by the same economic and social forcings as in any other group of people. The gap between the displayed speech and the actual behaviour is interpreted as the building of a specific spatial capital, which has close links with the type of space Foucault names "hétérotopie".

KEY WORDS : Bikes - Hétérotopie - Identity - Rennes - Spatial Capital. 
Dans la littérature scientifique anglo saxonne la moto et les motards sont objet d'intérêt. Les géographes anglophones qui s'intéressent à la moto, le font sous l'angle du genre, du sport ou de l'économie. Ils notent par exemple (Knigge, 2002) que les motards sont machos et passent plus de temps à louer la beauté de leur moto que celle de leur passagère. Ils signalent (Van Zuyle, 1988; Wezel et Lomi, 2003) l'importance économique des compétitions motos, et la domination technologique japonaise (Esbjornsson et al., 2005). Ils en étudient les aspects de sécurité routière (Reeder et al., 1995; Huang et Preston, 2004). En France, à notre connaissance les géographes ne s'intéressent pas à la moto. Aucune étude ne concerne les trajets, les itinéraires, les comportements des motards et donc leur rapport à l'espace.

L'idée qui motive cet article est que moto et motards peuvent être, en France, un objet d'étude capable de susciter des réflexions utiles à la géographie. Les motards français ont en effet quelques caractéristiques originales : ils revendiquent, au travers de plusieurs organisations, un usage particulier de l'espace public. Ils invoquent une sorte d'identité motarde qui devrait, selon eux, leur donner des droits particuliers dans le code de la route. Ils auraient un rapport à l'espace routier que nul ne peut comprendre s'il ne pratique pas la moto.

Nous proposons de tenter une approche géographique de ce discours en partant de la définition de «capital spatial » : «ensemble des ressources accumulées par un acteur lui permettant de tirer avantage, en fonction de sa stratégie, de l'usage de la dimension spatiale de la société » (Levy et Lussault, 2003). Les motards, rassemblés en association ayant monté leur propre système d'assurance (Marsetti, 2003) revendiquent leur capacité à conduire une moto comme une ressource, dont ils sont seuls possesseurs, et ils ont comme objectif de faire changer les règles qui les concernent (bridage des moteurs, remontée des files...). Cela leur procurerait un avantage : une sécurité plus grande dans la circulation. Cela leur fournirait un second avantage, qui est de l'ordre de la satisfaction hédoniste : ils auraient plus d'agrément à rouler. À tort ou à raison (mais ce n'est pas à nous d'en juger) ils revendiquent des privilèges dans l'usage d'une partie de l'espace public (les routes) à cause des spécificités de leur véhicule.

La problématique de notre article est donc la suivante : en quoi les motards ont-ils un usage particulier d'un espace commun, et qu'est ce que l'étude de leurs stratégies spatiales apporte à la réflexion théorique sur le concept de "capital spatial». Le plan de ce travail est justifié par le défaut de connaissances scientifiques au sujet des motards et de leurs pratiques de l'espace. Dans un premier temps, nous discutons des bases théoriques à partir desquelles nous pouvons construire un corpus (même réduit) d'informations. Il s'agit de réfléchir aux concepts qui peuvent orienter une enquête de terrain et guider le recueil de données. Les parties suivantes exploitent ces données, d'une façon qualitative d'abord, d'une manière quantitative ensuite.

\section{Les motards, leur capital spatial et leur hétérotopie}

Le capital spatial, s'il est défini comme en introduction fait appel à un cumul de compétences qui sont agencées stratégiquement pour un but précis. Une caractéristique générale, assez différente de la pratique automobile, est que les motards compensent les problèmes de friction spatiale par une mobilité choisie et pourvoyeuse de plaisir. Une interprétation plus précise de textes, de films, de journaux dans lesquels les motards sont mis en scène par des journalistes eux-mêmes motards, et souvent impliqués dans les actions de revendication peut être entreprise. Nous avons recensé les livres qui parlent de motos, les films et parcouru les magazines spécialisés afin d'en tirer quelques idées générales sur la façon dont les motards se décrivent eux-mêmes. Ces livres sont peu nombreux mais ciblent des thématiques très spécifiques et très différenciées d'un pays à l'autre. Nous en donnons quelques exemples.

Aux États-Unis, la marque Harley Davidson est associée, par le truchement de gangsters ouvertement nationalistes (Barger, 2000), à une idéologie de la domination des espaces vides par le parcours, cheveux au vent, de mécaniques civilisatrices prenant le relais des anciens chariots d’immigrants dans les plaines poussiéreuses du Mid-West. Toujours aux États-Unis, en parfaite 
opposition avec ce nationalisme, la moto est utilisée pour véhiculer des messages, des mythes, politiquement incorrects, avec Easy Rider (H. Fonda) ou The Wild One (M. Brando), ou avec le livre, dit « livre culte » qui s'intitule Traité du Zen et de l'Entretien des Motocyclettes (Pirsig, 1978). En Italie la moto est aussi l'objet d'une attention artistique et les fabricants demandent souvent à des artistes de dessiner des modèles nouveaux comme Philippe Starck pour Aprilla. Ces modèles ont le statut d'œuvre d'art et sont exposés dans des musées (Guggenheim, 1999; Suet et Thévenet, 2001). La moto est aussi un objet qui est politiquement à la mode. On réédite le récit que fait Guévara de son voyage, sur une moto anglaise peu fiable au travers des Andes (Che Guevara, 1997) et un film en est tiré. Un américain refait son trajet et écrit un best seller (Symmes, 2000).

En Angleterre la moto est un objet culturel hautement symbolique et terriblement ambivalent. Roulent à moto aussi bien des héros homosexuels (Lawrence d'Arabie, qui meurt dans un accident de moto) que des machos violents (dans Orange Mécanique), des explorateurs (Simon, 1979) ou des révolutionnaires adolescents (dans If). Les ouvriers de l'usine Triumph, de 1977 à 1981 s'organisent en coopérative autogérée et tentent une utopie gauchiste au début des années Thatcher (sans succès). La moto est un ingrédient essentiel du rock'n roll et des films d'action.

En France il existe des éléments d'image positifs. C'est par exemple la bande dessinée (avec la série Joe Bar Team) qui démontre que la moto est, au moins dans l'imaginaire des lecteurs, un enjeu significatif. C'est - autre exemple plus ancien - la chanson de variété qui fait de Brigitte Bardot et de sa Harley Davidson une image minutieusement construite, universellement séduisante. C'est aussi un art dit noble, la littérature qui attribue le prix Goncourt à l'auteur de La Motocyclette A. P. de Mandiargues (1963), récit érotique décrivant une jeune beauté sur une moto américaine parcourant des routes allemandes (et alsaciennes) pour joindre un amant français. C'est aussi une lutte sociale intense, puis une organisation durable qui permet à un mouvement de révolte conte la vignette des motos (projet giscardien) de se structurer et de construire une compagnie mutualiste d'assurance (la Mutuelle des Motards) qui, en 2005 assure toujours 160000 motos (Marsetti, 2003). En France la moto a aussi une forte dimension sportive mais il n'y a pas de diffusion des compétitions sur les chaînes hertziennes (et aucun impact médiatique) ce qui survalorise en contraste des films semi confidentiels comme Continental Circus ou Le Cheval de Fer (Laperrouzas, 1972).

Ce survol rapide permet de distinguer que, contrairement à bien des acteurs qui utilisent leur capital spatial pour en tirer un avantage non spatial, les motards utilisent leur capital spatial pour tenter de construire un espace "rêvé », qu'ils aimeraient parcourir sans contraintes. Ils imaginent une route "libre » de toutes limitations mais pourvoyeuse de rencontres, d'aventures et au-delà, de transgressions. Cette ambition se construit au travers des notions de ressources, de stratégie et se matérialise par des rassemblements humanitaires de motards.

La notion de ressource doit probablement être appréhendée au travers de ce que les motards appellent le pilotage. C'est une compétence qui leur est spécifique et qui est leur propriété exclusive. Seul un titulaire du permis moto sait piloter une moto. Il s'agit donc bien d'une ressource propre et c'est parce qu'ils l'utilisent que les motards revendiquent un usage spécifique de la route. Au nom des particularités du pilotage moto, ils exigent tels ou tels aménagements de sécurité. Ainsi les glissières de sécurité doivent être protégées par le bas afin qu’elles ne fonctionnent pas comme guillotine en cas de chute. Il est important de signaler que cette revendication a été jugée légitime par les gouvernements successifs et que ces équipements nouveaux sont progressivement mis en place partout. Cet exemple montre que la notion de ressource est non seulement exclusive, mais aussi un petit peu discriminatoire. Quelqu'un qui ne saurait pas piloter une moto n'aurait pas compétence pour juger du bien fondé de la revendication motarde. Là encore, les motards ont obtenu satisfaction : dans chaque département s'il y a un «monsieur moto », il doit avoir le permis moto.

La notion d'avantage (tirer avantage de l'usage de la dimension spatiale...) est beaucoup moins claire. Selon leurs propres dires les motards ne recherchent pas un avantage, ils recherchent le 
fait de rouler là où ils veulent, ce qui est un droit. Bien que cela ne soit pas explicitement dit, il apparaît aussi qu'ils cherchent à rouler à la vitesse qu'ils veulent et c'est probablement là qu'il faut chercher la notion de stratégie destinée à obtenir un avantage. La construction de l'argumentaire est toujours aussi simple et redondante : seul un motard sait piloter une moto, donc seul un motard sait juger de ce qui est dangereux ou pas. En conséquence le code de la route, conçu pour les voitures n'est pas adapté aux motos. De nombreux conflits actuels illustrent cet enjeu (feux de jour, droit de remonter une file, stationnement sur le trottoir, etc.). Pour les motards, la ressource pilotage est invoquée comme devant mener à un avantage : un code de la route spécialement aménagé pour eux.

La notion de stratégie est très claire. Selon la définition l'avantage obtenu est «selon sa stratégie » c'est-à-dire qu'il sert un objectif propre à l'acteur. La stratégie utilisée doit même être pensée comme mobilisant les compétences (les ressources) accumulées. Dans de nombreux cas, pour obtenir un avantage, des groupes sociaux agissent par lobbying. Les motards européens le font aussi, soutenus par des constructeurs européens comme BMW, Triumph ou Ducati qui sont opposés aux limitations de vitesses comme au bridage des moteurs. Mais cette stratégie-là ne leur est pas particulière. Les motards, en France (faute d'un constructeur français qui pourrait aider au lobbying, mais sur une idée de Coluche) ont donc inventé un type d'action spectaculaire qui est destiné à leur conférer une image de gens responsables, altruistes, et donc... d'interlocuteurs incontournables pour toute politique de sécurité routière. Les rassemblements humanitaires, les « relais calmos », les encadrements de manifestations sportives, sont des outils pour contribuer à la fabrication d'une image de responsabilité. Le plus ancien et le plus vaste rassemblement motard pour Les Restos du Cœur a lieu à Rennes.

Le rassemblement des « Motards ont du Cœur » nous paraît être un exemple intéressant. La réunion de motos représente toutes les couches de la société, et représente une action que la société définit comme bonne : lever des fonds pour les mal nourris. Mais la société est aussi contestée puisque ce que font Les Restos du Cœur est ce que la société n'est pas capable de faire : nourrir tout le monde. L'inversion vient de ce que ce sont les motards (ceux qui sont, statistiquement les plus responsables de la « violence routière ») qui sont les plus solidaires et les plus généreux... il y a renversement par rassemblement des contraires. Les motards, pour l'occasion endossent pour leur tenue de cuir la plus « rebelle » et viennent afficher une dégaine de voyous pour mieux faire ressortir leur humanitarisme. Ils y portent volontiers une tenue voyante qui n'est pas celle avec laquelle ils rouleraient s'ils devaient faire trois cents kilomètres... Tout le monde a le droit de rentrer pour regarder les motos, mais seuls motards et passagères peuvent participer au défilé. Toutes les motos sont soigneusement parquées en ordre, toutes, ensuite, roulent par deux de front à $60,70 \mathrm{~km} / \mathrm{h}$ maximum, et toutes se garent de nouveau en ordre sur une place de Rennes après un défilé de 70 kilomètres.

Le capital spatial des motards est donc une construction savante : un usage modéré de la route (peu de kilomètres en moyenne) mais un usage spectaculaire de l'espace public afin de construire une image qui donnera aux associations de motards du poids dans les négociations de politique sécuritaire. Pour cela, les motards français ont inventé un « lieu-événement » original (unique au monde à l'origine mais maintenant mis en place en Belgique, en Espagne...) qui est le grand rassemblement humanitaire de motards. Le concept qui parait le plus adéquat pour le comprendre est alors celui d’hétérotopie. Il a été inventé par Foucault (édition 2001) qui l'explique comme étant un emplacement proche d'une utopie. Il écrit que ce sont des «sortes d'utopies effectivement réalisées dans lesquelles les emplacements réels, tous les autres emplacements réels que l'on peut trouver à l'intérieur de la culture sont à la fois représentés, contestés et inversés, des sortes de lieux qui sont hors de tous les lieux, bien que pourtant ils soient effectivement localisables ».

"L’hétérotopie a le pouvoir de rassembler en un seul lieu plusieurs espaces qui sont eux-mêmes incompatibles ", elle est " en rupture absolue avec le temps traditionnel », elle suppose un système « qui l'isole ou la rend pénétrable », mais au prix « d'un rite » et elle crée un ordre spatial « aussi méticuleux, aussi bien arrangé que le nôtre (l'espace non hétérotopique) est désordonné, mal agencé et brouillon » 
Les motards, durant cette réunion, montrent un comportement déguisé, qui n’est ni leur tenue habituelle, ni leur vitesse habituelle, ni leur conduite habituelle. Il s'agit d'un défilé de parade qui donne à voir les motards comme ce qu'ils ne sont pas (toujours) mais comme ils souhaitent être vus, afin d'être écoutés et si possible, politiquement satisfaits. Le terme hétérotopie prend alors un sens riche : un lieu qui est l'autre, dans l'espace et dans le temps, des lieux habituels des acteurs spatiaux. Dans le capital spatial des motards il y a une ressource qui n'est pas seulement la capacité à user de l'espace, mais aussi la capacité à inventer un espace et une mise en scène concomitante.

Si notre hypothèse au sujet du capital spatial et de l'hétérotopie est pertinente, nous devrions pouvoir observer une différence assez significative entre ce que des motards disent d'eux-mêmes lors d'une réunion comme Les Restos du Cœur et leurs comportements quotidiens, en dehors de ce rassemblement. Nous avons donc décidé de profiter de notre participation à une de ces réunions pour nous y livrer à une sorte d’observation en temps réel. Nous avons tenté de discerner s'il existait bien, comme notre hypothèse le suppose, deux choses distinctes : un discours motard identitaire d'une part, un comportement motard plus banal d'autre part. Il s'agit alors de recenser ce que les participants disent d'eux-mêmes à cette occasion. Mais nous avons aussi construit un questionnaire qui oblige le motard à donner des informations sur son comportement habituel.

\section{Méthodologie : enquêtes et entretiens}

Les enquêtes ont été faites avec une grille. Elles ont été réalisées en juin 2004. C'était la plus grosse concentration de motos à but humanitaire en Europe cette année-là. Cependant elle ne draine que 2500 motos et ne concerne que des motards locaux, très majoritairement rennais. Il s'agit donc d'un échantillon assez spatialement homogène pour qu'une étude de leur déplacement fasse ressortir des constantes imputables à la localisation, ou des différences qui sont explicables par des raisons autres (sociales, familiales). Lors de cette ballade le questionnaire d'enquête a été proposé à - environ - une moto toutes les vingt motos, au moment où elles arrivent pour se rassembler. Quelques questionnaires supplémentaires ont été remplis dans les jours suivant par internet. Tous les interrogés, sauf deux, sont rennais ou des environs de Rennes. Quatre-vingts questionnaires complètement remplis ont été recueillis, et une cinquantaine d'autres, incomplets n'ont pas été exploités de façon systématique. Ils ont cependant servi d'un point de vue qualitatif.

Les enquêtes ont été complétées par des entretiens semi-directifs (par reformulation) ou non directifs. Tous (un peu plus d'une cinquantaine) ont été menés de « motard à motard » l'enquêteur se présentant d'abord comme motard(e), ensuite (éventuellement) comme géographe. Ces entretiens ont eu lieu après qu'un traitement statistique rapide ait fait ressortir quelques points intéressants au sujet des destinations les plus fréquentes, de l'usage (solo ou duo) de la moto et du type de la machine (japonaise, italienne, allemande, etc.). Les entretiens ont donc ciblé des motards(es) rennais(es) de telle façon que les proportions observées lors des Restos du Cœur soient respectées. Les entretiens ont eu lieu, en 2004 et 2005 lors de concentrations touristiques des environs de Rennes, lors de Porcaro, lors de concentrations humanitaires à Nantes, Rennes et à Vannes, lors de réunions sur circuits (Carole en Île de France, Le Vigeant au Poitou, Le Mans, Fougères, etc.). Certains entretiens (effectués sur les embarcadères de Saint-Malo, Cherbourg et Roscoff) ont spécifiquement ciblé des Rennais partant passer des vacances motos en Angleterre, en Irlande, au pays de Galles et à l'île de Man. Dans tous les cas, d'autres que des Rennais ont été interrogés, mais à but de comparaison. 


\section{Les motards, leurs stratégies de déplacement, vus et racontés par eux-mêmes}

Cette partie présente l'aspect qualitatif du dépouillement de nos enquêtes et entrevues. Notre position épistémologique, en partie inspirée par les réflexions de B. Collignon (2005), est de prendre les discours tenus par les motards comme vrais, sans chercher à les décrypter avec une clé de lecture sociale. Nous rendons compte en premier lieu des propos tenus le plus souvent dans les entretiens, qui peuvent être classés dans trois grandes thématiques : l'esthétique de la moto, la qualité des routes, les pratiques de groupe des motards. Dans un second temps nous rendons compte des réponses données à des questions semi-directives portant sur la nature du plaisir éprouvé à moto.

Le discours spontané est celui qui s’instaure entre deux motards qui se rencontrent à l'occasion d'une réunion du type Resto du Cœur. Il s'organise le plus souvent autour de trois axes majeurs. Le premier et le plus important est celui de la notion de belle moto. L'objet, l'esthétique, le tuning, sont les premiers thèmes que l'on aborde pour faire connaissance. Une fois que la moto est décrite (admirée) la conversation tourne autour de la notion de performance, de vitesse. Enfin, dans les minutes suivantes, les motards parlent de la route qu'ils ont suivie pour arriver là et s'attardent à décrire les virages les plus intéressants, ils abordent la notion de pilotage. Ces discours impliquent toujours une critique forte des limitations et des radars. Ils débouchent toujours sur des points de vue très revendicatifs

Le discours spontané aborde aussi d'autres thèmes, mais dans un deuxième temps. Il semble qu'après qu'on ait dit qu'on roule vite, on puisse dire qu'on sait aussi rouler doucement et regarder le paysage. La notion d'itinéraire entre alors dans la conversation. Il ne s'agit pas de décrire la route parcourue, mais d'expliquer pourquoi on a choisi cette route plutôt qu'une autre. Presque toujours les motards disent choisir des "petites routes » et éviter les 4 voies. Cependant, sous la pluie, et s'ils utilisent leur moto pour aller au travail, ils empruntent la 4 voie plutôt que les " glissantes » petites routes. La présence de la passagère est parfois mentionnée dans la conversation, mais ce n'est jamais le premier thème ni celui auquel le plus de temps est consacré. Cette remarque reprend des observations faites aux États-Unis par L. D. Knigge (2002) qui mentionne que les bikers attachent autant d'importance (voire plus!) à l'esthétique de leur moto qu'à celle de leur passagère. Les contraintes de temps, d'argent, voire de famille (enfants) sont parfois mentionnées «si on avait plus de temps, on ferait... ». Ce discours est variable suivant l'âge. Il en existe une version jeune, qui insiste sur les contraintes, en particulier financières et qui a des propos relativement conformistes sur les destinations (schématiquement c'est «la ballade à la mer»). Dans la version tenue par des motards plus vieux on distingue plus d'attention à l'itinéraire et des destinations plus variées. Un peu plus d'attention est portée au confort de la passagère. Un troisième aspect du discours porte sur ce que les motards évoquent comme activité associée à la moto, et il s'agit très souvent d'action humanitaire (rassemblement pour la mucoviscidose, la myopathie, ballades pour handicapés mentaux, encadrement et sécurisation des parcours pour des courses de vélo ou des marathons).

Tous ces discours ne disent jamais pourquoi on pratique la moto, ni quel plaisir on y prend. Ces deux notions sont implicites (dans la notion de pilotage par exemple) mais jamais exprimées ni explicitées. Elles font donc l'objet d'une partie des questions semi-directives.

Les motards, dans l'ensemble ont très peu d'arguments rationnellement exprimables pour expliquer leur « passion ». Il semble, à les écouter, qu’il est évident que si on fait de la moto c'est qu'on aime ça et que la raison pour laquelle on l'aime n'a aucune importance, ou qu'elle relève d'une évidence non exprimable. Les termes invoqués sont essentiellement les suivants : liberté (au sens d'échapper aux embouteillages et de sortir de la ville en un clin d'œil), vitesse et précision de la trajectoire, sport de plein air, moteur coupleux, moteur puissant, ce qui n'est pas synonyme de grande vitesse mais d'accélération en sortie de courbe. La destination de la ballade est rarement invoquée comme raison de plaisir, sauf pour se rendre à une compétition. Les motards ont 
beaucoup plus d'arguments pour décrire la route sur laquelle ils pilotent avec plaisir. Les termes invoqués le plus souvent sont ceux qui concernent la sécurité : type d'enrobé, granulométrie du bitume, continuité/raccord, évacuation d'eau, bandes peintes, glissières, éventuel gazole en rondpoint... Une deuxième série de termes concerne les virages : courbure, dévers, visibilité, planitude, dégagement, enchaînement, et surtout, échanges d'informations sur telle portion de route où l'on peut « ouvrir grand » pour passer « le premier à deux dix et prendre le suivant à deux vingt cinq... et prendre les virolos suivants entre cent quarante, cent cinquante. ». Il s'agit d'un discours très fortement ressemblant à la bande dessinée du Joe Bar Team et il est très fréquent. Il arrive aussi que la route soit belle à cause du paysage qu'elle traverse. Chez les interrogés, la beauté du paysage n'est jamais associée à un itinéraire fréquent mais à un itinéraire de week-end ou de vacances qui est toujours, soit en montagne, soit en bord de mer. Aucun motard interrogé n'a jamais dit qu'une ville pouvait constituer, à moto un beau paysage, alors que tous disent apprécier de s'y réunir pour prendre des pots.

À des interrogations précises sur la nature du plaisir de conduire, les motards apportent parfois des réponses précises. Elles font apparaître les notions de défoulement (sortir du boulot et ouvrir en grand!), d'évasion (se distraire du quotidien, sortir de son cadre) et font ressortir l'importance de la passagère (nous deux sans personne...) avec qui une relation amoureuse privilégiée est toujours présente. C'est aussi un des rares moments ou les passagères interviennent : elles aiment cela parce qu'elles « aiment le mec » et il en résulte qu'elles cherchent à passer leur permis (et le possèdent pour un bon tiers d'entre elles). Nous n'avons vu qu'une seule fois une femme conduire et un homme passager : c'était pour une suspension de permis. Les questions demandant de réfléchir à la spécificité de la moto procurent des réponses dans le même domaine : évasion, liberté, vitesse. Les questions sur le choix des destinations entraînent deux types de réponses. Dans un premier temps les propos tenus sont : " On va partout, on va où on veut, on sait pas toujours où on va quand on part... » Ce qui est cohérent avec les notions de liberté et d'évasion. Si la question n'est plus «où tu vas? », mais « et alors t'arrives où? », les destinations sont beaucoup plus précises : "On arrive là où on a de la famille qui accueille, ou on arrive au bord de la mer. » La même question concernant des vacances à moto a une réponse négative dans beaucoup de cas (les vacances ne sont pas à moto). Dans le cas de réponse positive, les destinations sont très variables mais excluent les destinations nordiques, sauf dans les îles britanniques et, ponctuellement de l'Allemagne et de la Belgique. Les vacances à motos se conçoivent d'abord au soleil (Portugal, Grèce, Croatie, etc.). Un dernier groupe de question porte sur ce que l'on aime pas à moto : les réponses sont immédiates : les radars, les «bleus » (gendarmes et policiers), puis la pluie, le verglas, le gazole, les téléphones portables des automobilistes...

Une synthèse rapide de ces propos, considérés comme vrais, permet de décrire comment le motard cherche à se faire percevoir d'un autre motard (Lacombe, 1970). Il dit être : prudent et passionné de vitesse, esthète en mécanique, libre, quasiment toujours en couple, convivial, généreux...

Il est important de constater qu'il y a des points qu'il n'aborde jamais : son statut social, ses revenus, son automobile (tous en ont une), ses opinions politiques (sauf contre la répression routière), la pollution atmosphérique.

Le motard se voit (ou se décrit) donc en tant que motard, comme un être mobile par choix (Ollivro, 2005), dégagé de contraintes sociales banales, et original par son souci de liberté et de générosité. Comment ce portrait s'accommode-t-il de ce que, froidement, les chiffres disent du comportement de ces mêmes motards?

\section{Les motards, leurs inscriptions sociales et spatiales, décrites par des chiffres}

L'échantillonnage compte 71 questionnaires exploitables quantitativement. L'analyse des traitements statistiques a permis de valider des résultats attendus, mais a aussi révélé des comporte- 
ments, des phénomènes plus surprenants, remettant en cause certaines idées préconçues au sujet des populations motardes. Nous abordons d'abord quelques correspondances statistiques simples, puis nous exposons les résultats d'une AFC qui permet de construire quatre classes.

L'analyse des fréquences et des corrélations fait ressortir plusieurs points. La moto est avant tout une histoire d'hommes à près de $86 \%$. Le choix de la cylindrée, s'il peut être très diversifié pour les hommes, demeure très limité pour les femmes qui possèdent très souvent de petites cylindrées (600 $\mathrm{cm}^{3}$ en moyenne). Cela étant, ces chiffres ne reflètent que partiellement la participation des femmes dans la pratique de la moto puisqu'elles sont très souvent passagères : près de $51 \%$ des enquêtés passent plus de la moitié de leur temps de conduite en duo; dans ces cas, et pour des besoins de confort de conduite et même de sécurité, la cylindrée de la moto est logiquement assez élevée. Les motos japonaises dominent très largement l'échantillon avec près de 85 \% des motos interrogées; viennent ensuite les européennes avec $14 \%$; les américaines, une fois n'est pas coutume, sont très largement minoritaires avec un peu plus de $1 \%$. Sur la France entière (avec des chiffres qui incluent les scooters; Orluc, 2006) on compterait autour de 27,5\% d'européennes, $3 \%$ d'américaines, autour de $60 \%$ de japonaises et le reste en autres motos asiatiques (coréennes, indiennes et chinoises). En ce qui concerne les cylindrés, notre échantillon n'est pas, non plus, représentatif de la moyenne française. En France les $1000 \mathrm{~cm}^{3}$ et plus font 11,9 et 12,4\% (en 2004 et 2005) des immatriculations neuves. Dans notre échantillon elles font près de $40 \%$, ce qui laisse à penser que nombre d'entre elles sont des motos d'occasion.

La distribution des classes d'âge des motards souligne un éventail de population très diversifié, avec une surreprésentation de la classe d'âge des 40-49 ans qui regroupe à elle seule $40 \%$ des enquêtés. L'âge moyen est de 37 ans. Les contraintes familiales étant nettement allégées à partir de cette décennie (vis-à-vis des enfants), le pouvoir d'achat étant théoriquement plus important, la pratique de la moto devient (beaucoup de conducteurs adultes ont depuis peu leur permis : coefficient de corrélation de 0.574 ) ou redevient possible (reprise de la moto après un temps d'arrêt). En France l'âge moyen du passage du permis moto est estimé à 36 ans. Notre enquête s'accommode assez bien de cette valeur (fig. 1). La moto n'est donc plus seulement une affaire de jeunes, de même que la cylindrée de la moto. Le stéréotype jeune/moto puissante, adulte/moto plus «sage » est à revoir. La corrélation révèle une très grande indépendance entre ces critères ; d'ailleurs les motos les plus puissantes rencontrées au cours de l'enquête ont des propriétaires qui frisent la cinquantaine. Cela étant, il ne faut pas confondre puissance de la moto et prise de risque en matière de conduite : le respect des vitesses est beaucoup plus ignoré chez les jeunes

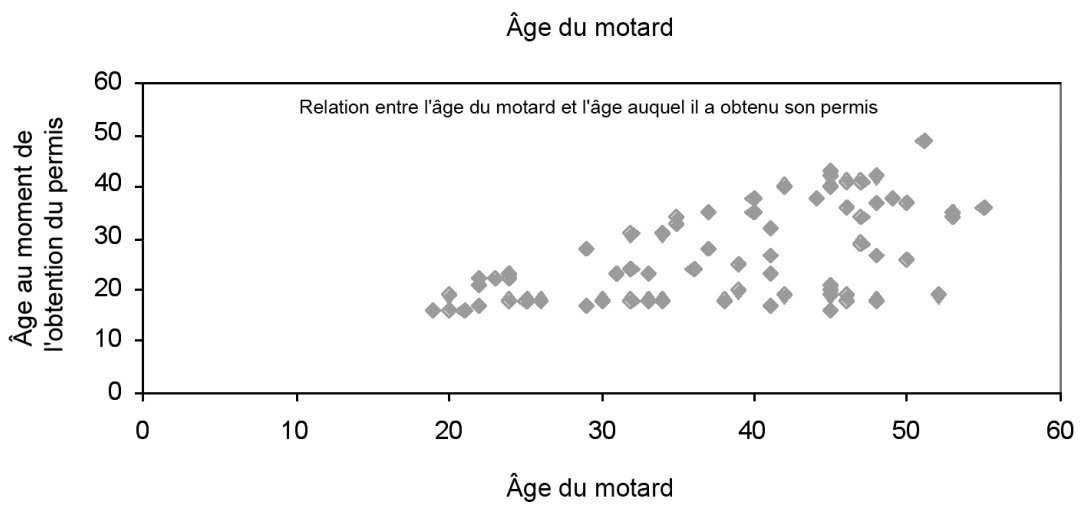

Figure 1: Nombre de motards obtiennent leur permis vers 20 ans. D'autres, dans cet échantillon, viennent d'obtenir le leur. Enfin une troisième population est entre les deux Many bikers achieve their licence at (about) 20. Others, in this graph, have just achieved it. Some are in between 
qui déclarent souvent dépasser largement voire très largement les limitations de vitesse (taux de corrélation inverse).

Le profil social des motards interrogés est assez singulier puisqu'une large domination des employés/ ouvriers est observée : près de $50 \%$ des enquêtés. Les catégories dites supérieures approchent tout juste $17 \%$ et les professions intermédiaires regroupent environ $35 \%$ des motards. Le profil global de la société française ne se retrouve donc pas dans celui de la population motarde. Par ailleurs, la prise en compte de l'âge du motard apporte une importante nuance analytique : une certaine corrélation existe entre les motards jeunes qui sont plutôt ouvriers / employés et les motards plus âgés qui appartiennent plus souvent aux catégories dites supérieures. Il n'existe pas d'enquête comparable au niveau national et nous ne savons pas si cette population motarde est spécifique à Rennes ou pas. L'utilisation de la moto, souvent envisagée comme un objet de liberté pour les temps de congés, est finalement beaucoup plus diversifiée. Elle a souvent un statut de véhicule utilitaire pour les déplacements pendulaires quotidiens : $42 \%$ des usagers l'utilisent tous les jours sur des distances comprises entre 5 et $40 \mathrm{~km}$ qui correspondant à des déplacements intra-urbains et périurbains. Dans les temps de congés, la moto redevient un objet de loisirs pour les ballades de fin de semaine et les virées de vacances. En affinant les résultats, on s'aperçoit que la moto est avant tout un « outil » de week-end et que son utilisation pour les vacances (supposant de plus grandes distances à parcourir) reste finalement assez marginale : seuls $30 \%$ des enquêtés l'utilisent pendant les périodes de vacances. Ce résultat était assez peu attendu car il va à l'encontre de l'idée de motards se déplaçant sur de grandes distances, dans de grands espaces, avec une grande liberté de mouvement. Finalement, la moto a ses contraintes (fatigue, équipements embarqués, facteurs climatiques)... et celles-ci semblent l'emporter très largement sur ses atouts.

Les déplacements des motards rennais pour le week-end révèlent deux faits marquants du point de vue géographique (fig. 2). Très logiquement, les déplacements s’inscrivent dans un espace pro-

Figure 2 : Destinations de week end. Le Mans domine à cause des compétitions Week end trips: Le Mans leads because of motorcycle races

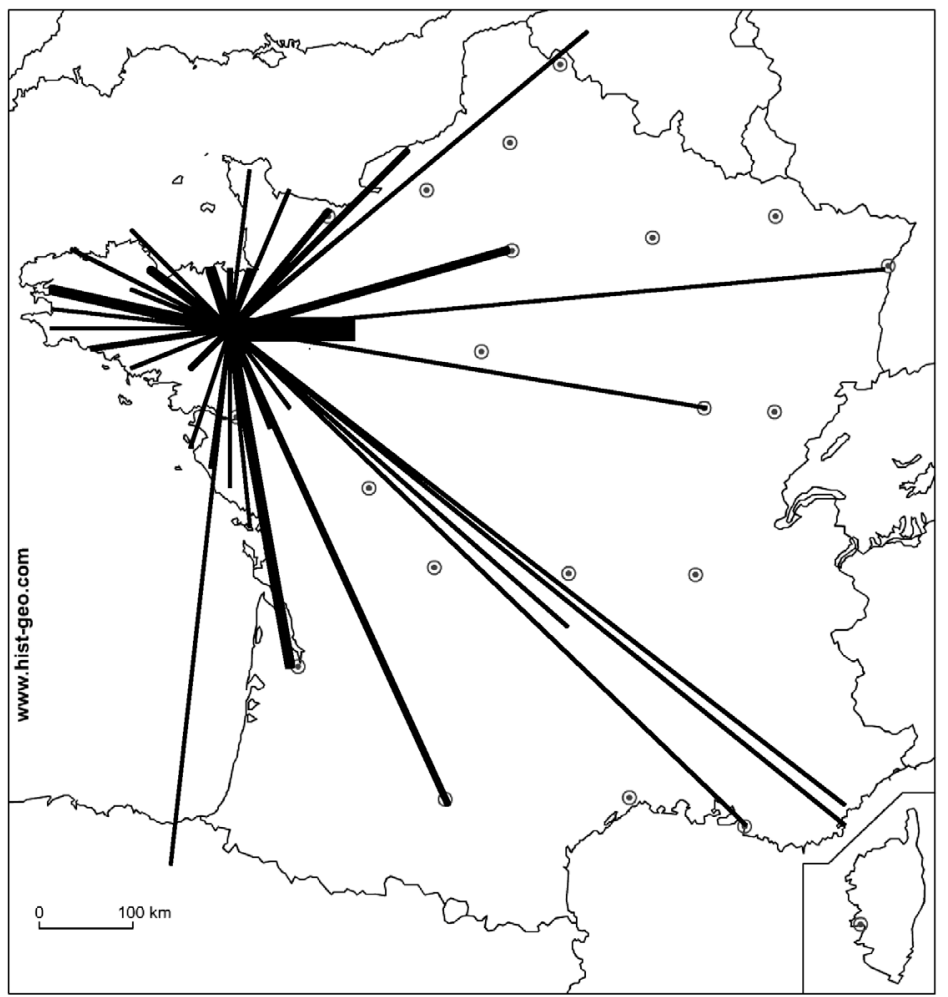


che : le faisceau en étoile autour de Rennes privilégie la Bretagne (et ses littoraux) qui demeure la destination privilégiée, de même que les côtes vendéennes et Le Mans (en raison du nombre d'organisations événementielles autour de la moto et de l'automobile). Sur les plus grandes distances, les villes restent des pôles de destination et d'attractivité majeurs (Dijon, Bordeaux, Strasbourg, Paris, Toulouse, etc.). Finalement, les mobilités spatiales dessinent un arc s'étirant du nord au sud de la France en passant par les côtes de la Manche et de l'Atlantique. Certaines régions sont ignorées, mais une enquête complémentaire montrerait sans aucun doute de nouvelles destinations tout en confirmant les traits majeurs qui ressortent de la carte.

L'analyse factorielle des correspondances permet de comprendre l'organisation de l'information. Les trois premiers axes de l'AFC regroupent un peu plus de $27 \%$ de l'information totale, avec un plan principal totalisant environ $20 \%$. On s'attend donc à ne disposer que d'une assez faible structuration de l'information statistique globale, confirmant la grande diversité de situation possible déjà perceptible par l'analyse des corrélations simples (tableau 1).

\begin{tabular}{|c|c|c|c|c|c|c|c|}
\hline & Sexe & Âge motard & $\begin{array}{l}\text { Nationalité } \\
\text { de la moto }\end{array}$ & Profession & $\begin{array}{c}\text { Année } \\
\text { d'obtention } \\
\text { du permis }\end{array}$ & $\begin{array}{c}\text { Âge au } \\
\text { moment de } \\
\text { l'obtention } \\
\text { du permis }\end{array}$ & $\begin{array}{l}\text { Temps de } \\
\text { pratique en } \\
\text { duo }\end{array}$ \\
\hline Âge motard & 0.076 & & & & & & \\
\hline Nationalité de la moto & 0.153 & -0.342 & & & & & \\
\hline Profession & -0.104 & 0.458 & -0.322 & & & & \\
\hline Année d'obtention du permis & 0.160 & -0.565 & 0.296 & -0.410 & & & \\
\hline $\begin{array}{l}\text { Âge au moment de l'obten- } \\
\text { tion du permis }\end{array}$ & 0.281 & 0.574 & -0.062 & 0.090 & 0.332 & & \\
\hline Temps de pratique en duo & -0.367 & -0.055 & -0.069 & 0.039 & -0.103 & -0.145 & \\
\hline Cylindrée & -0.419 & 0.174 & -0.274 & 0.183 & -0.308 & -0.108 & 0.482 \\
\hline Âge de la moto & -0.030 & 0.041 & -0.015 & 0.026 & -0.165 & -0.140 & 0.005 \\
\hline Km annuel & -0.296 & -0.154 & -0.145 & 0.001 & -0.107 & -0.236 & 0.060 \\
\hline Km journalier & -0.193 & -0.158 & 0.081 & 0.038 & 0.079 & -0.084 & 0.042 \\
\hline Km Week-end & -0.078 & 0.243 & -0.122 & 0.318 & -0.249 & 0.035 & 0.117 \\
\hline Km Vacances & -0.039 & 0.122 & -0.014 & 0.154 & -0.089 & 0.104 & 0.015 \\
\hline Vitesse & -0.207 & -0.311 & 0.016 & -0.180 & 0.068 & -0.317 & 0.001 \\
\hline
\end{tabular}

\begin{tabular}{|l|r|r|r|r|l|l|}
\hline & \multicolumn{1}{|l|}{ Cylindrée } & Âge de la moto & Km annuel & Km journalier & $\begin{array}{l}\text { Km } \\
\text { Week-end }\end{array}$ & $\begin{array}{l}\text { Km } \\
\text { Vacances }\end{array}$ \\
\hline Âge de la moto & -0.046 & & & & & \\
\hline Km annuel & 0.226 & -0.186 & & & & \\
\hline Km journalier & -0.030 & -0.047 & 0.366 & & & \\
\hline $\begin{array}{l}\text { Km } \\
\text { Week-end }\end{array}$ & 0.108 & 0.175 & 0.100 & 0.067 & & \\
\hline $\begin{array}{l}\text { Km } \\
\text { Vacances }\end{array}$ & 0.154 & -0.356 & 0.242 & -0.026 & 0.015 & \\
\hline Vitesse & 0.030 & 0.071 & 0.232 & 0.042 & -0.104 & -0.085 \\
\hline
\end{tabular}

Tableau 1 : Matrice des corrélations simples Matrix of simple correlations

L'axe 1, axe des abscisses, (10,43\% de l'information) est largement structuré par les classes d'âge et les catégories socioprofessionnelles; il oppose, du côté des coordonnées négatives, les jeunes motards (moins de trente ans) ayant obtenu leur permis depuis moins de 5 ans, circulant sur de petites cylindrées (moins de $600 \mathrm{~cm}^{3}$ ) et appartenant à des milieux ouvriers et employés, et, du côté des coordonnées positives, les « vieux » motards (plus de quarante ans et ayant obtenu 
leur permis dans les années 1970-1980), appartenant aux catégories socioprofessionnelles dites supérieures et utilisant leur moto sur de grandes distances les fins de semaine. La moto est donc avant tout un objet de loisir dans cette configuration. Il est intéressant de constater que l'on ne retrouve pas associées à ces modalités les grosses cylindrées.

L'axe 2, axe des ordonnées, (9,54 \% de l'information totale) est structuré non pas par des modalités opposantes (variable de type 1 contre type 3), mais plutôt par des groupes de modalités décrivant des profils comportementaux extrêmement distincts. Ainsi dans sa partie négative, l'axe 2 se structure autour de modalités représentatives d'une catégorie de population motarde bien connue dans les référents du grand public : profil type du jeune motard (de moins de 30 ans et qui a obtenu son permis entre 18 et 20 ans) totalement investi dans la pratique de la moto; laquelle est à la fois objet utilitaire et de loisirs sur de grandes distances (aussi bien quotidiennement qu'en fin de semaine). Grand rouleur qui recherche les sensations en se jouant des limitations de vitesse. Dans sa partie positive, on retrouve une population plus «sage » composée essentiellement de femmes qui pratiquent la moto exclusivement en solo et qui sont devenues conductrices tardivement (entre 30 et 49 ans, période d'allégement des contraintes familiales). La pratique de la moto reste toutefois occasionnelle : elles roulent généralement très peu aussi bien au quotidien qu’à l'année.

L'axe 2 distingue donc deux types de conducteurs dont les profils et les attentes diffèrent considérablement en matière de pratique de conduite. Une classification hiérarchique ascendante, qui combine la signification des deux premiers axes et projette les individus sur le plan principal, permet de construire une sorte de typologie, bien que le nombre d'individus par classe soit réduit.

La classe 1, la plus importante en effectifs (près de $44 \%$ des individus), pourrait être qualifiée de «classe aux profils moyens ». On y rencontre plutôt des hommes, mais aussi des femmes (étirement vers le haut de l'axe 2) autour de la trentaine et issus des CSP intermédiaires. Ces conducteurs d'âges mûrs, qui roulent majoritairement sur des japonaises de cylindrées moyennes, en solo et occasionnellement en duo, ont généralement acquis une certaine expérience, les incitant à rester raisonnables et prudents sur la route (très peu d'accidents). La moto reste avant tout un objet de plaisir pour les fins de semaine, l'utilisation quotidienne reste marginale.

La classe 2 (24\% de l'effectif total), est essentiellement composée d'hommes dans la trentaine et la quarantaine issus des CPS supérieures. Ce sont généralement de « vieux et de grands rouleurs » aussi bien pendant la semaine, les week-ends et les vacances. En France une moto parcours en moyenne $6000 \mathrm{~km}$ par an. Dans cette catégorie la moyenne est supérieure à $11000 \mathrm{~km} / \mathrm{an}$. Les motos sont de nationalités fort différenciées (pas de "déterminisme japonais ») et d'assez grosses cylindrées. Par contre, on remarquera qu'il s'agit d'une classe où les accidents corporels et matériels sont plus fréquents.

La classe 3 (21\% des individus), à cause de sa localisation sur le plan principal, reprend et croise en grande partie des profils décrits du côté des coordonnées négatives des axes 1 et 2 : population masculine et jeune, appartenant à des milieux ouvriers et employés, et qui roulent essentiellement en japonaises de petites cylindrées (faute certainement de moyens financiers suffisants pour envisager mieux). Jeunes conducteurs peu enclins à respecter les limitations de vitesses malgré une utilisation intensive de la moto, notamment sur l'année et au quotidien (assez peu les fins de semaine).

Enfin, la classe 4, (seulement $11 \%$ des effectifs, soit 8 individus) apparaît comme une catégorie un peu à part, même si elle présente des points concomitants avec la classe précédente. On y retrouve des jeunes qui ont obtenu le permis très tôt (aux alentours de 18 ans) et qui roulent sur de grosses cylindrées, régulièrement (notamment au quotidien), mais aussi beaucoup en duo. Malgré cette relative jeunesse en matière d'expérience de conduite et l'utilisation de moto puissantes, il semble que l'on ait affaire à des personnes plus raisonnables, ou en tout cas plus attentives en 
matière de conduite, puisque les accidents corporels et matériels sont assez peu fréquents (en supposant bien entendu que les enquêtés aient répondu avec honnêteté aux questions).

Pour résumer cette analyse quantitative, on retient la grande diversité des profils motards (fig. 3). Les populations motardes rennaises participant aux « Restos du Cœur » ne reprennent pas le profil global de la société. La moto touche et interpelle tout le monde, mais selon des pratiques et des finalités extrêmement variables. L'âge, le sexe et l'expérience apparaissent comme des critères très déterminants dans les choix et les comportements routiers. Il n'y a donc pas une identité motarde, mais des pratiques de la moto qui sont conditionnées par le rapport au quotidien et donc à l'espace du quotidien (trajets et horaires en particulier). Envisagée comme un objet de liberté, de loisir, la moto est avant tout un objet utilitaire pour citadins et reste fortement tributaire de nombreuses contraintes d'ordre familial, climatique, financière, technique et même «imaginaires ", limitant son utilisation sur de longues distances et de longues durées. Finalement, les motards apparaissent comme des gens « comme tout le monde », pas du tout en marge de la société (même si certains se revendiquent comme tel) et soumis aux mêmes contraintes et aléas que tout un chacun. En quoi les motards peuvent-ils prétendre à quoique ce soit de spécifique dans leur usage de l'espace?

Cela semble pouvoir nous permettre de valider notre hypothèse de départ : les motards utilisent leur « capital spatial » pour obtenir un avantage qu’ils veulent spécifique... mais leur usage de la route ne semble pas très différent de celui qu'en font d'autres personnes, dans les mêmes conditions de contraintes familiales et financières. L'image qu'ils veulent donner d'eux-mêmes dans un rassemblement est donc, sinon fausse, du moins artificielle, construite pour l'occasion et relève plus du mythe que du réel. Le rassemblement humanitaire a donc des caractères d'hétérotopie et la spécificité de l'identité motarde pourrait n’être qu'illusion. Cependant la spécificité de la moto, en tant que véhicule, subsiste parce qu'elle a un équilibre différent de celui d'une voiture. En ce sens les motards peuvent revendiquer une spécificité d'ordre technique. Ils utilisent le même espace que les automobilistes, à peu prés selon les mêmes contraintes semble-t-il, mais avec un instrument différent. Cela incite à réfléchir au fait qu'il n'y pas d'usage de la dimension spatiale de la société sans un outil, un instrument qui rend cet usage possible. Tous les véhicules ne sont pas, sur ce plan, équivalents.

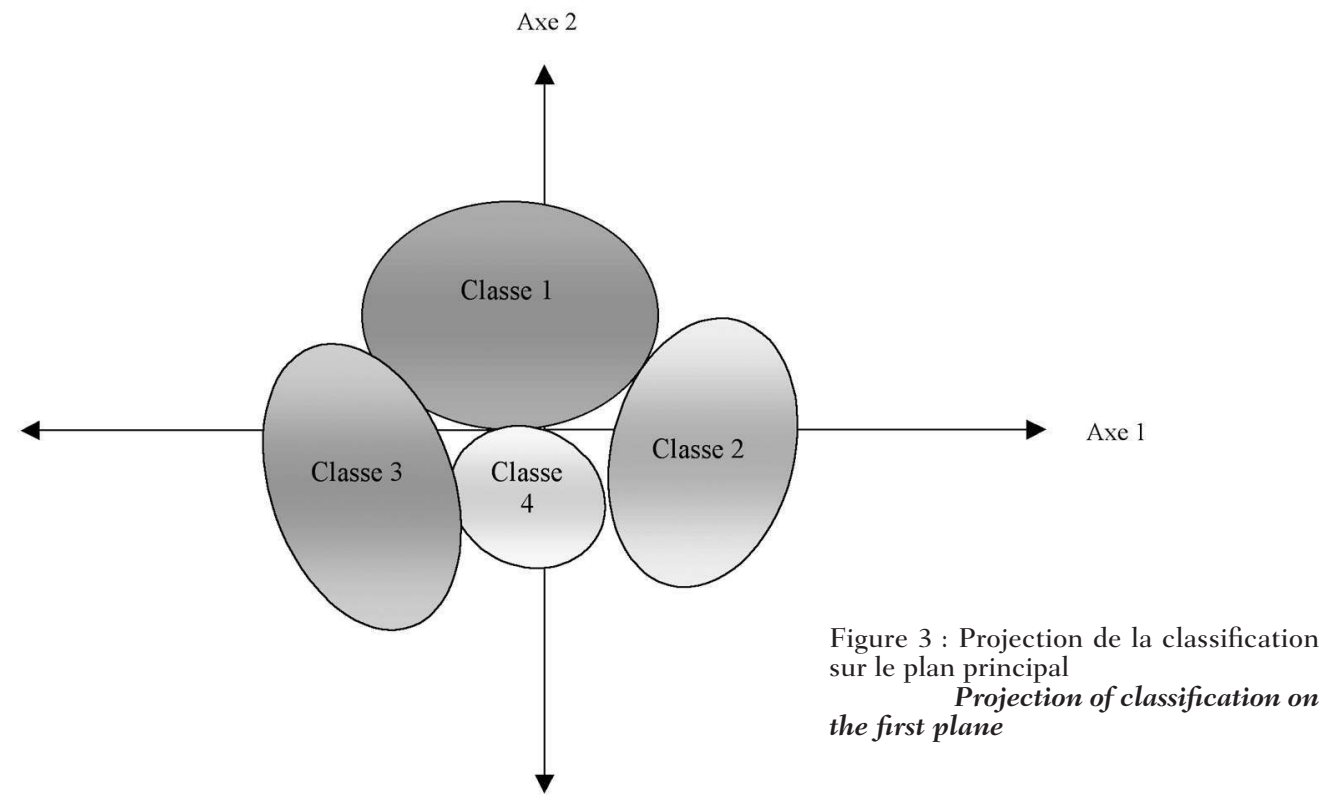


L'étude des motards soulève un paradoxe dans la notion de capital spatial. Comme nous l'avons vu, ce concept rassemble les notions de ressource, stratégie et avantage. Si l'on appréhende chacune d'elles au travers du filtre de l'objet technique qui permet le déplacement on peut envisager - au moins - deux types de comportements logiques et contradictoires. Un premier type de logique repose sur l'analyse des caractéristiques propres à chaque moyen de transport. Chaque véhicule a ses avantages en tel lieu, ses inconvénients en tel autre. La stratégie spatiale optimale est alors de disposer, à chaque instant, du type de véhicule adéquat. Une conception rationnelle du capital spatial pourrait alors entraîner chaque groupe humain à alterner d'un type de transport à l'autre, selon les circonstances et les buts. Dans ce cas le plus important dans le concept de capital spatial est probablement la notion de stratégie. Nous pouvons qualifier cette logique par les termes de «rationalité multimodale»

Un deuxième type de logique repose sur l’idée qu'un véhicule particulier est (arbitrairement et subjectivement) plus agréable que tous les autres, en toutes circonstances. De ce point de vue l'usage d'un véhicule est appréhendé comme un plaisir spécifique lié à ses caractères techniques. A partir de ce plaisir, une sorte de « culture » (illusion) se construit et est intériorisée par les acteurs. Certains motards sont convaincus d'être différents. De ce fait ce qu'ils pensent être un raisonnement rationnel n'obéit pas à des critères logiques qui pousseraient à l'alternance des moyens de transport. Ils invoquent, par exemple, le fait de n'avoir que deux roues et d'occuper peu d'espace sur la chaussée, pour justifier l'emploi, en ville, de la moto au lieu des transports en commun. Pour eux, le capital spatial serait probablement appréhendé avec un accent fort sur la notion de ressource. Leur logique peut alors être dite « subjective et monomodale».

Les résultats de l'AFC semblent indiquer que les motards de la classe 1 ont plutôt une logique « rationnelle », ouverte à la multimodalité tandis que ceux des classes 2, 3 et 4 ont plutôt une logique « subjective » moins attentive à la multi modalité.

\section{Conclusion}

Une approche superficielle du monde de la moto fait penser que l'usage que les motards font de l'espace public (la route) est modeste en durée mais intense à cause de la vitesse qu'ils pratiquent. Dans cette optique le nombre élevé de motards tués est un problème de vitesse excessive. Une analyse plus fine montre qu'il n'y a pas un usage mais des usages, qui sont différents selon l'age, le sexe, le revenu, et qui, dans l'ensemble, sont très fortement contraints par des facteurs sociaux économiques peu originaux. Les motards sont, un peu comme tout le monde, mais ils sont aussi un peu différents : ils savent en particulier qu'ils vivent une passion plus dangereuse qu'une autre. Pour faire entendre ce qu'ils pensent être une revendication spécifique (identitaire?) quant à leur sécurité, les motards ont inventé une forme particulièrement originale de mise en oeuvre de leur capital spatial. Au lieu de simplement mobiliser leurs ressources, leurs compétences spatiales pour obtenir un avantage, ils inventent un lieu hors norme, un lieu carnavalesque de spectacle et d'inversion des conduites, une hétérotopie dans laquelle, quelques fois par an, ils exhibent une image telle qu'on ne peut que les trouver sympathiques.

Cette stratégie spatiale est paradoxale : les motards sont très différents les uns des autres, mais en construisant de toute pièce l'image d'une seule population généreuse, ils encouragent les spectateurs à ne pas discerner leurs différences. Quand des chiffres disent qu'il meurt 6 à 10 fois plus de motards que d'automobilistes (par kilomètre parcouru), il est possible que les politiques ne cherchent pas à distinguer entre de bons et de mauvais pilotes, et qu'ils continuent à prendre des mesures globales et, il faut aussi le reconnaître, parfois inadaptées.

Cette stratégie spatiale a aussi l'intérêt d'inciter les géographes à réfléchir aux concepts par lesquels ils appréhendent la capacité de groupes sociaux à user de l'espace. Le concept de capital spatial est, sur ce plan, particulièrement efficace. Il convient cependant de l'enrichir en s'interrogeant sur l'éventuelle importance de la dimension technique du véhicule utilisé et sur la dimension identitaire qui guide le choix qu'on en fait. 


\section{Bibliographie}

Collignon (B.), 2005. - «Que sait-on des savoirs géographiques vernaculaires? », BAGF, n³, p. 321 331.

Esbjornsson (M.), Juhlin (O.), Ostergren (M.), 2005. - « Traffic encounters and Hocman associating motorcycle ethnography with design », Mobility Interactive Institute, Stokholm, sp Pub, p. 1-18.

Foucault (M.), 2001. - Dits et Écrits, II, 1976-1988, Paris, Gallimard, 1736 p.

Huang (B.), Preston (J.), 2004. - «A litterature review on motorcycle accidents », Transport studies Unit, Final Report, University of Oxford, $52 \mathrm{p}$.

Knigge (L. D.), 2002. - "The Sturgis motorcycle rally: Public Space, Identiy and Gender », American Association of Geographers, 98th Annual Meeting, Abstracts, p. 210-212.

Levy (J.), Lussault (M.), 2003. - Dictionnaire de la géographie et de l'espace des sociétés, Paris Belin, $1034 \mathrm{p}$.

Ollivro (J.), 2005. - «Les classes mobiles », L'information géographique, 69, 3, p. 28-44.

Reeder (A. I.), Chalmers (D. J.), Langley (J. D.), 1995. - « Young on-road motorcyclists in New Zealand: age of licensure, unlicenced riding, and motorcycle borrowing », Injury Prevention, 1, p. 103-108.

VAn ZuYLE (P.), 1988. - « Battle Line in the Desert. A Report on the Barstow-to-Vegas Motorcycle Race, and the California Desert », Sport Place, Journal of Sports Geography, Vol. 2, n 1, p. 15-21.

WeZEL (F. C.), LOMi (A.) 2003. - « The organizational advantage of nations: An ecological perspective on the evolution of the motorcycle industry in Belgium, Italy and Japan, 1894-1993 », dans BAUm (J. A. C.), Sorenson (O.) (ed.), Geography and Strategy, Greenwich CT, JAI Press, coll. «Advances in Strategic Management, 20 », p. 359-392.

\section{Sources}

Barger (S.), 2000. - Hell's Angel, the life and times of Sonny Barger and the Hell's Angel Motorcycle Club, Fouth Estate, $260 \mathrm{p}$.

Che Guevara (E.), 1997. - Voyage à Motocyclette, Éditions Mille et une Nuits, 224 p.

De Mandiargues (A. P.), 1963. - La Motocyclette, Paris, Gallimard, 212 p.

Guggenheim 1999. - El Arte de la Motocicleta, Guggenheim Museum Publications, 434 p.

Lacombe (C.), 1970. - La Moto, Planète, 231 p.

Laperrousaz (J.), 1972. - Continental Circus, Paris, Solar, 218 p.

MarsetTi (M.), 2003. - Chronique d'une utopie en marche : la mutuelle des motards, Éditions Fédération Française des Motards en Colère, 216 p.

Orluc (P.), 2006 . - « Le marché français a fait exploser les compteurs en 2005 », Moto Journal, n 1696, p. 8-9.

Pirsig (R.-M.), 1978. - Traité du Zen et de l'entretien des motocyclettes, Points, 447 p.

Simon (T.), 1979. - Jupiter's Travels, Penguin, 447 p.

Suet (B.), Thévenet (J.-M.), 2001. - Des motos et des hommes, Éditions Eden/Janvier, non paginé.

Symmes (P.), 2000. - Chasing Che, Robinson, 302 p.

Cet article a été reçu le 26 mai 2006 et définitivement accepté le 21 septembre 2007. 